\title{
In-vitro anti-inflammatory activity, free radical (DPPH) scavenging, and ferric reducing ability (FRAP) of Sargassum cristaefolium lipid-soluble fraction and putative identification of bioactive compounds using UHPLC-ESI-ORBITRAP-MS/MS
}

\begin{abstract}
Sargassum brown seaweed is well-known to contain several bioactive compounds which exhibit various biological activities, including anti-inflammatory and antioxidant activity. Lipophilic extracts and fractions of Sargassum were reported to possess promising anti-inflammatory activity. This study, therefore, aims to evaluate the anti-inflammatory and antioxidant activity of Sargassum cristaefolium crude lipid extract and its fractions. The brown seaweed was obtained from Awur Bay, Jepara - Indonesia. Crude lipid fractionation was performed using normal phase column chromatography, and three different fractions (dichloromethane, acetone, methanol) were produced. The results showed that treatment of acetone fraction exerted strongest nitric oxide inhibition in lipopolysaccharide-induced RAW 264.7 cells, both in pre-incubated and co-incubated cell culture models. This outcome was in accordance with its 2,2-diphenyl-1-picrylhydrazyl (DPPH) scavenging activity and ferric reducing antioxidant power (FRAP). Metabolite profiling of lipid fractions was performed by ultra-high-performance liquid chromatography electrospray ionization orbitrap tandem mass spectrometry, while the orthogonal projection to latent structures analysis was conducted to determine some features with significant correlation to the bioactivity. There were 14 feature candidates considered from both positive and negative ionization mode datasets. Seven out of them were putatively identified as pheophytin a (1), all-trans fucoxanthin (2), 132-hydroxy-pheophytin a (3), pheophorbide a (4), 1-hexadecanoyl-2-(9Z-octadecenoyl)-3O- $\beta$-D-galactosyl-sn-glycerol (6), 1-(5Z,8Z,11Z,14Z,17Z-eicosapentaenoyl)-2-(9Z,12Z,15Zoctadecatrienoyl)-3-O- $\beta$-D-galactosyl-sn-glycerol (10), and 1-(9Z,12Z,15Z-octadecatrienoyl)-2(6Z,9Z,12Z,15Z-octadecatetraenoyl)-3-O- $\beta$-D-galactosyl-sn glycerol.
\end{abstract}

Keyword: Brown seaweed; FRAP; Functional lipid; Nitric oxide; DPPH radical scavenging; RAW 264.7 macrophage cell 